\title{
Rotating hyperdeformed states in light nuclear systems
}

\author{
G. Royer, J. Gaudillot \\ Laboratoire Subatech, UMR: IN2P3/CNRS-Université-Ecole des Mines, Nantes 44, France
}

\begin{abstract}
The existence of rotating quasi-molecular hyperdeformed states formed in the entrance channel of capture reactions of light nuclei is predicted within a rotational liquid drop model including the nuclear proximity energy. The $l$-dependent capture barrier heights and positions as well as the angular momentum, the energy and the moment of inertia ranges of these very deformed high spin states are given for the reactions ${ }^{13} \mathrm{C}+{ }^{13} \mathrm{C},{ }^{16} \mathrm{O}+{ }^{16} \mathrm{O},{ }^{28} \mathrm{Si}+{ }^{12} \mathrm{C},{ }^{28} \mathrm{Si}+{ }^{16} \mathrm{O},{ }^{24} \mathrm{Mg}+{ }^{24} \mathrm{Mg},{ }^{28} \mathrm{Si}+{ }^{24} \mathrm{Mg}$, ${ }^{28} \mathrm{Si}+{ }^{28} \mathrm{Si},{ }^{28} \mathrm{Si}+{ }^{40} \mathrm{Ca},{ }^{40} \mathrm{Ca}+{ }^{40} \mathrm{Ca},{ }^{40} \mathrm{Ca}+{ }^{48} \mathrm{Ca},{ }^{48} \mathrm{Ca}+{ }^{48} \mathrm{Ca}$ and ${ }^{58} \mathrm{Ni}+{ }^{58} \mathrm{Ni}$. Analytical formulas are provided for any reaction between light nuclei.
\end{abstract}

PACS numbers: 25.70.Jj,21.10.Re,24.10.-i

\section{INTRODUCTION}

The connection between nuclear clustering, quasi-molecular resonances, orbiting phenomena and super and hyperdeformations in light and medium nuclear systems remains a fruitful domain in nuclear physics [1]. Binary and ternary decays of rotating hyper-deformed states formed in the ${ }^{32} \mathrm{~S}+{ }^{24} \mathrm{Mg}$ and ${ }^{36} \mathrm{Ar}+{ }^{24} \mathrm{Mg}$ reactions at angular momenta around $50 \hbar$ have been observed recently $[2,3]$. Resonances observed in the excitation functions of some reactions between light nuclei as well as the numerous detected superdeformed bands are also indications of the existence of rotating deformed stabilized configurations in the ${ }^{36} \mathrm{Ar},{ }^{40} \mathrm{Ca},{ }^{44} \mathrm{Ti},{ }^{48} \mathrm{Cr}$ and ${ }^{56} \mathrm{Ni}$ nuclei [4-9]. For heavier nuclear systems the situation is less clear since evidence of the formation of high spin hyperdeformed structures in the ${ }^{37} \mathrm{Cl}+{ }^{120} \mathrm{Sn}$ and ${ }^{51} \mathrm{~V}+{ }^{100} \mathrm{Mo}$ reactions has been advanced $[10,11]$ before concluding that there is inconsistency with the properties of a band structure [12]. For these masses it had been rather assumed that very high spin states would not be populated since the fission decay mode would be dominant. Nevertheless, rotational structures with high moments of inertia have been identified in the ${ }^{76} \mathrm{Ge}+{ }^{76} \mathrm{Ge}$ and ${ }^{28} \mathrm{Si}+{ }^{124} \mathrm{Sn}$ reactions at spin around $65 \hbar[13,14]$. The new generation of $\gamma$ detectors could perhaps allow to elucidate this problem. Furthermore, for these heavier nuclear systems the hindrance to complete fusion is a phenomenon presenting in the most part of the capture events due to the onset of the quasifission process which competes with complete fusion during the evolution of the composed system formed at capture stage and requires theoretically the framework of combined dynamical and statistical models $[15-17]$.

In the present work the purpose is to study the entrance channel of reactions between light nuclei and to focus on high angular momenta, the fission probability being much smaller for such light systems. The approach leading to the prediction of hyperdeformed medium mass nuclear systems at very high spins [18] has been used again. The selected quasi-molecular one-body shape sequence (elliptic lemniscatoids) describes smoothly the formation of a deep neck between the incoming spherical nuclei while keeping almost spherical ends. The $l$-dependent fusion barriers are determined within a generalized liquid drop model [19-21]. For heavier nuclear systems the introduction of the deformation of the separated nuclei and of the angle between their symmetry axes may lower the potential barriers and change their width and then change the capture cross sections $[15,22]$.

\section{GENERALIZED LIQUID DROP MODEL}

The generalized liquid drop model (GLDM) includes the volume, surface, Coulomb and proximity energies :

$$
E=E_{V}+E_{S}+E_{C}+E_{\text {Prox }} .
$$

When the nuclei are separated:

$$
\begin{gathered}
E_{V}=-15.494\left[\left(1-1.8 I_{1}^{2}\right) A_{1}+\left(1-1.8 I_{2}^{2}\right) A_{2}\right] \mathrm{MeV} \\
E_{S}=17.9439\left[\left(1-2.6 I_{1}^{2}\right) A_{1}^{2 / 3}+\left(1-2.6 I_{2}^{2}\right) A_{2}^{2 / 3}\right] \mathrm{MeV} \\
E_{C}=0.6 e^{2} Z_{1}^{2} / R_{1}+0.6 e^{2} Z_{2}^{2} / R_{2}+e^{2} Z_{1} Z_{2} / r
\end{gathered}
$$


where $A_{i}, Z_{i}, R_{i}$ and $I_{i}$ are the mass numbers, charge numbers, radii and relative neutron excesses of the two nuclei. $r$ is the distance between the mass centres. The radii $R_{i}$ are taken as

$$
R_{i}=\left(1.28 A_{i}^{1 / 3}-0.76+0.8 A_{i}^{-1 / 3}\right) \mathrm{fm} .
$$

For one-body shapes, the surface and Coulomb energies are defined as:

$$
\begin{gathered}
E_{S}=17.9439\left(1-2.6 I^{2}\right) A^{2 / 3}\left(S / 4 \pi R_{0}^{2}\right) \mathrm{MeV} \\
E_{C}=0.6 e^{2}\left(Z^{2} / R_{0}\right) \times 0.5 \int\left(V(\theta) / V_{0}\right)\left(R(\theta) / R_{0}\right)^{3} \sin \theta d \theta .
\end{gathered}
$$

$S$ is the surface of the one-body deformed nucleus. $V(\theta)$ is the electrostatic potential at the surface and $V_{0}$ the surface potential of the sphere.

The surface energy results from the effects of the surface tension forces in a half space. When there are nucleons in regard in a gap or a neck between incoming nuclei or separated fragments an additional term called proximity energy must be added to take into account the effects of the nuclear forces between the close surfaces,

$$
E_{\text {Prox }}(r)=2 \gamma \int_{h_{\min }}^{h_{\max }} \Phi[D(r, h) / b] 2 \pi h d h .
$$

$h$ is the distance varying from the neck radius or zero to the height of the neck border. $D$ is the distance between the surfaces in regard and $b=0.99 \mathrm{fm}$ the surface width. $\Phi$ is the proximity function. The surface parameter $\gamma$ is the geometric mean between the surface parameters of the two nuclei. This term is essential to describe smoothly the two-body to one-body transition and to obtain reasonable fusion barrier heights. The barrier top corresponds to two still separated nuclei and the pure Coulomb barrier is strongly flattened and reduced. It has been previously shown that the combination of this GLDM and of the selected quasi-molecular shape sequence allows to reproduce both the fusion barrier heights and radii, the fission, the $\alpha$ decay and the cluster radioactivity data [23, 24].

\section{CAPTURE BARRIERS}

The $l$-dependent capture barriers of twelve reactions between light nuclei are displayed in Fig. 1 and the Q value and the barrier heights and positions are given in Table 1. The experimental barrier heights and positions are derived from the fusion cross sections and depend on the selected attractive potential to describe the potential barriers and several values are often given in the literature for the same reaction with sometimes important error bars. However that may be the GLDM allows to reproduce satisfactorily the whole data set. With increasing angular momenta a macroscopic very deformed minimum appears and corresponds to a one-body shape with a deep neck. Thus the formation of hyperdeformed quasi-molecular states is predicted in a large angular momentum range. Often the beam energy is not sufficient to reach the quasi-spherical compound nucleus assuming the angular momentum conservation which ensures a relative stability of these highly deformed rotating states and prevents from a direct compound nucleus formation. In this approach the quasi-fission exit channel is neglected and the capture events lead to these quasi-molecular hyperdeformed states and then are fusion events at very high angular momenta. A sub-barrier energy is probably the best way to form these exotic configurations. A similar conclusion has been drawn in ref. [25]. The deformation of these minima increases with the angular momentum while the position of the barrier is closer to the contact point. Due to a more important mass inertia the maximal angular momenta that the nuclei are able to sustain increase with their masses. For these highly deformed shapes the calculations of the shell and pairing effects are very model dependent. They can move the position of these potential pockets but their basic origin is macroscopic and, consequently, the existence of these hyperdeformed states must be a general phenomenon for light nuclei for which the fission channel is relatively narrow.

\section{FUSION CROSS SECTIONS}

To test the validity of the GLDM the fusion cross sections have been determined using the usual partial-wave summation

$$
\sigma_{f u s}(E)=\pi \star^{2}(E) \sum_{l}(2 l+1) T_{l}(E)
$$


TABLE I: Experimental Q values of the studied fusion reactions and experimental and theoretical barrier heights and positions.

\begin{tabular}{ccccccc}
\hline \hline Reaction & $Q_{\exp }(\mathrm{MeV})$ & $B_{\exp }(\mathrm{MeV})$ & $R_{\exp }(f m)$ & $B_{G L D M}$ & $R_{G L D M}$ & Ref. \\
\hline${ }^{13} \mathrm{C}+{ }^{13} \mathrm{C} \rightarrow{ }^{26} \mathrm{Mg}^{*}$ & 22.47 & 6.0 & 7.83 & 5.85 & 8.1 & {$[26]$} \\
${ }^{16} \mathrm{O}+{ }^{16} \mathrm{O} \rightarrow{ }^{32} \mathrm{~S}^{*}$ & 16.54 & 10.25 & 8.21 & 10.3 & 8.2 & {$[26]$} \\
${ }^{28} \mathrm{Si}+{ }^{12} \mathrm{C} \rightarrow{ }^{40} \mathrm{Ca}^{*}$ & 13.35 & 13.7 & 8.0 & 13.2 & 8.4 & {$[27]$} \\
${ }^{28} \mathrm{Si}+{ }^{16} \mathrm{O} \rightarrow{ }^{44} \mathrm{Ti}^{*}$ & 11.32 & 17.7 & 8.2 & 17.3 & 8.55 & {$[27]$} \\
${ }^{24} \mathrm{Mg}+{ }^{24} \mathrm{Mg} \rightarrow{ }^{48} \mathrm{Cr} *$ & 14.95 & 21.5 & 8.4 & 21.9 & 8.7 & {$[28]$} \\
${ }^{28} \mathrm{Si}+{ }^{24} \mathrm{Mg} \rightarrow{ }^{52} \mathrm{Fe} *$ & 12.91 & 25.9 & 8.5 & 25.2 & 8.8 & {$[27]$} \\
${ }^{28} \mathrm{Si}+{ }^{28} \mathrm{Si} \rightarrow{ }^{56} \mathrm{Ni}^{*}$ & 10.92 & 28.7 & 9.06 & 29.0 & 8.95 & {$[26]$} \\
${ }^{28} \mathrm{Si}+{ }^{40} \mathrm{Ca} \rightarrow{ }^{68} \mathrm{Se}^{*}$ & -2.19 & - & - & 40.2 & 9.25 & - \\
${ }^{40} \mathrm{Ca}+{ }^{40} \mathrm{Ca} \rightarrow{ }^{80} \mathrm{Zr}^{*}$ & -14.44 & 55.6 & 9.1 & 55.6 & 9.6 & {$[29]$} \\
${ }^{40} \mathrm{Ca}+{ }^{48} \mathrm{Ca} \rightarrow{ }^{88} \mathrm{Zr}^{*}$ & 04.56 & 53.2 & 10.1 & 54.0 & 9.9 & {$[30]$} \\
${ }^{48} \mathrm{Ca}+{ }^{48} \mathrm{Ca} \rightarrow{ }^{96} \mathrm{Zr}^{*}$ & -2.99 & 51.7 & 10.4 & 52.4 & 10.2 & {$[30]$} \\
${ }^{58} \mathrm{Ni}+{ }^{58} \mathrm{Ni} \rightarrow{ }^{116} \mathrm{Ba}^{*}$ & -65.86 & 102.5 & 10.2 & 100.6 & 10.4 & {$[27]$} \\
\hline \hline
\end{tabular}

where $\mathrm{E}$ is the centre-of-mass energy and $\lambda$ is the reduced de Broglie wavelength of the incident ion. Above the $l$-wave barrier the transmission coefficient is approximated by the Hill-Wheeler formula [26]. Below the barrier the WKB method has been used :

$$
T_{l}=\left[1+\exp \left(2 K_{l}\right)\right]^{-1}
$$

with

$$
K_{l}=\int_{r_{a}}^{r_{b}}\left[\frac{2 \mu}{\hbar^{2}}(E(l, r)-E)\right]^{1 / 2} d r
$$

$r_{a}$ and $r_{b}$ denote the classical turning points of the $l$-wave barrier at the center-of-mass energy. The Fig. 2 displays six fusion cross sections around the Coulomb barrier. The agreement is quite correct which indicates that the static approach is sufficient for these relatively light systems when the fusion barriers are precisely determined, even if for the light ${ }^{16} \mathrm{O}+{ }^{16} \mathrm{O}$ system reported in Fig. 2(a) the results of the calculation appear just acceptable. The quasi-fission events being neglected all the angular momenta leading to hyperdeformed rotating states contribute to the fusion cross sections which consequently are highly dependent on the angular momentum range. As for most one-dimensional static models using spherical shapes the theoretical fusion cross sections are slightly too low at very low energy below the barrier for some reactions. Several explanations have been advanced to explain this fusion cross section enhancement at sub-barrier energies : dynamic barrier fluctuation caused by orientational and vibrational fluctuation of the surface-tosurface distance, deformations, coupled channel effects, two-dimensional barrier transparency,... . For heavier masses dynamical models are necessary to reproduce the data since the top of the barrier is closer to the contact point and the dissipation due to the friction forces must be taken into account [19].

\section{CHARACTERISTICS OF THE ROTATING HYPERDEFORMED STATES}

In Tables II and III the angular momentum, moment of inertia and energy range of these highly deformed rotating quasi-molecular states are given for symmetric and asymmetric reactions as well as the $l$-dependent barrier heights and positions and the electric quadrupole moment and $\beta$ parameter in the symmetric case. The state corresponding to a potential pocket is taken into account when there is a barrier height of at least two MeV both against decay in two fragments and in the path towards the quasi-spherical compound nucleus. The $\beta$ parameter indicates clearly that the deformation is very large. Naturally these states probably will evacuate their excitation energy via $\gamma$ cascades and will reach after the superdeformed minima mainly due to shell effects at smaller deformations. It is interesting to note the high dependence on the isotope mass in the $\mathrm{Ca}+\mathrm{Ca}$ reactions due to the difference in the mass inertia and Q-values.

The following analytical formula reproduces the energy of the quasi-molecular minima versus its angular momentum with a rms deviation of $\sigma=0.69 \mathrm{MeV}$

$$
\begin{aligned}
& E=-11.18 A_{1}^{2 / 3}-16.025 A_{2}^{2 / 3}+\frac{50.618 A_{1}^{1 / 3} A_{2}^{1 / 3}}{A_{1}^{1 / 3}+A_{2}^{1 / 3}}+1.7624 A+7.9856 l^{0.5} \\
& +\frac{1.05985\left(A_{1}+A_{2}\right) l^{2}}{A_{1} A_{2}\left(A_{1}^{1 / 3}+A_{2}^{1 / 3}-2\right)}-\frac{0.20305 Z_{1}^{2}}{A_{1}^{1 / 3}}+\frac{0.06709 Z_{2}^{2}}{A_{2}^{1 / 3}}+\frac{1.13156 Z_{1} Z_{2}}{A_{1}^{1 / 3}+A_{2}^{1 / 3}-2},
\end{aligned}
$$



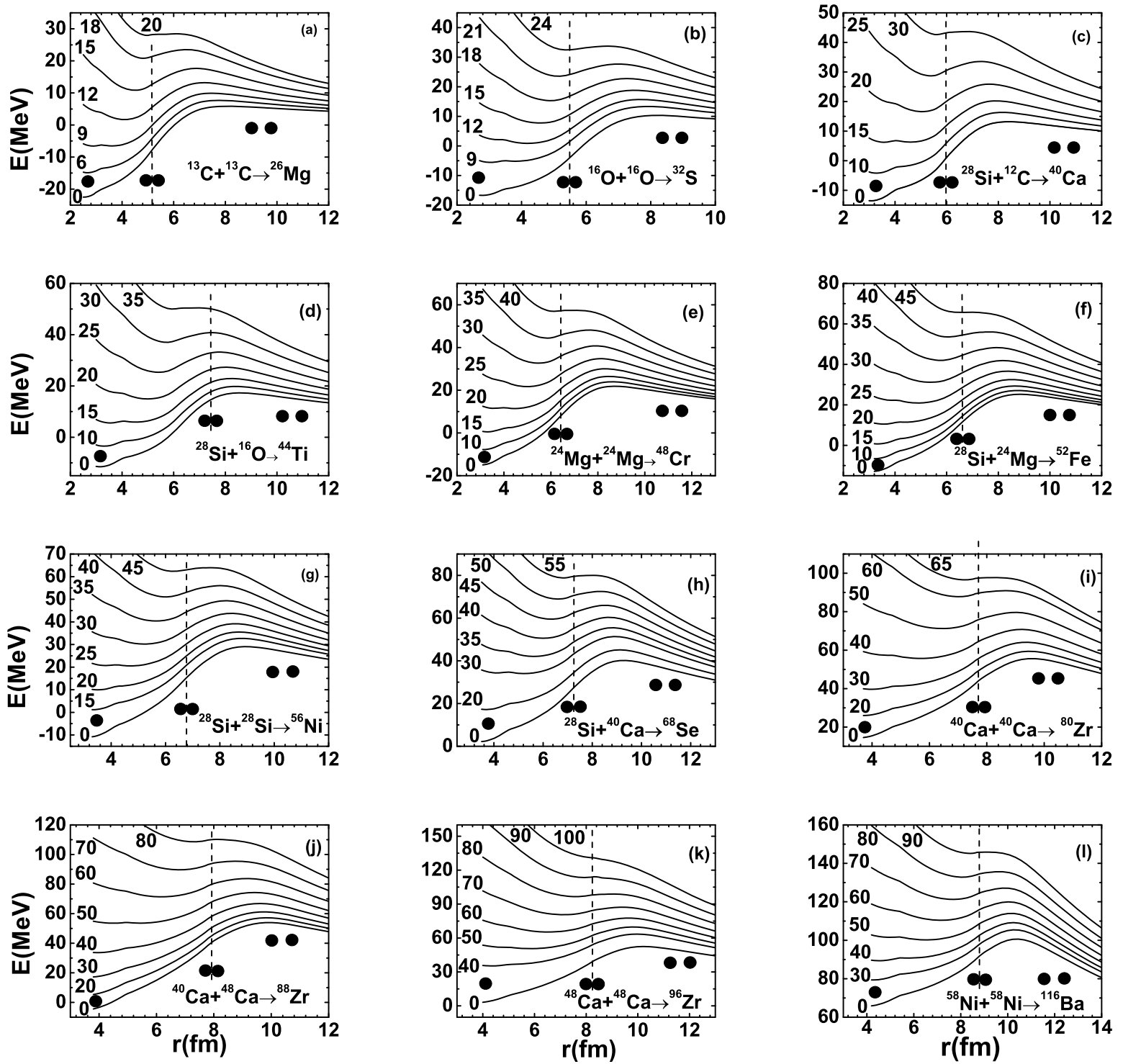

FIG. 1: Fusion barriers versus the angular momentum ( $\hbar$ unit) and the distance $\mathrm{r}$ between the mass centers for the ${ }^{13} \mathrm{C}+{ }^{13} \mathrm{C}$, ${ }^{16} \mathrm{O}+{ }^{16} \mathrm{O},{ }^{28} \mathrm{Si}+{ }^{12} \mathrm{C},{ }^{28} \mathrm{Si}+{ }^{16} \mathrm{O},{ }^{24} \mathrm{Mg}+{ }^{24} \mathrm{Mg},{ }^{28} \mathrm{Si}+{ }^{24} \mathrm{Mg},{ }^{28} \mathrm{Si}+{ }^{28} \mathrm{Si},{ }^{28} \mathrm{Si}+{ }^{40} \mathrm{Ca},{ }^{40} \mathrm{Ca}+{ }^{40} \mathrm{Ca},{ }^{40} \mathrm{Ca}+{ }^{48} \mathrm{Ca},{ }^{48} \mathrm{Ca}+{ }^{48} \mathrm{Ca}$ and ${ }^{58} \mathrm{Ni}+{ }^{58} \mathrm{Ni}$ nuclear systems. The dashed vertical line indicates the contact point between the spherical nuclei.

while the moment of inertia of the deformed state corresponding to the highest possible angular momentum is given by

$$
I=0.032966 \frac{A_{1} A_{2}\left(A_{1}^{1 / 3}+A_{2}^{1 / 3}-2\right)^{2}}{A}+0.0775167 A+0.0029512 A^{2}-0.00001253 A^{3}+3.75 * 10^{-8} A^{4},
$$

with $\sigma=0.29 \hbar^{2} / \mathrm{MeV}$.

For completeness the $l$-dependent capture barrier heights and positions are given in Table IV when there is no hyperdeformed macroscopic minimum. They can be useful for studies relative to superdeformed states.

\section{SUMMARY AND CONCLUSION}

The $l$-dependent capture barriers in reactions between light nuclei have been determined within a generalized liquid drop model taking into account the proximity energy and the asymmetry. Deep strongly deformed quasi-molecular 

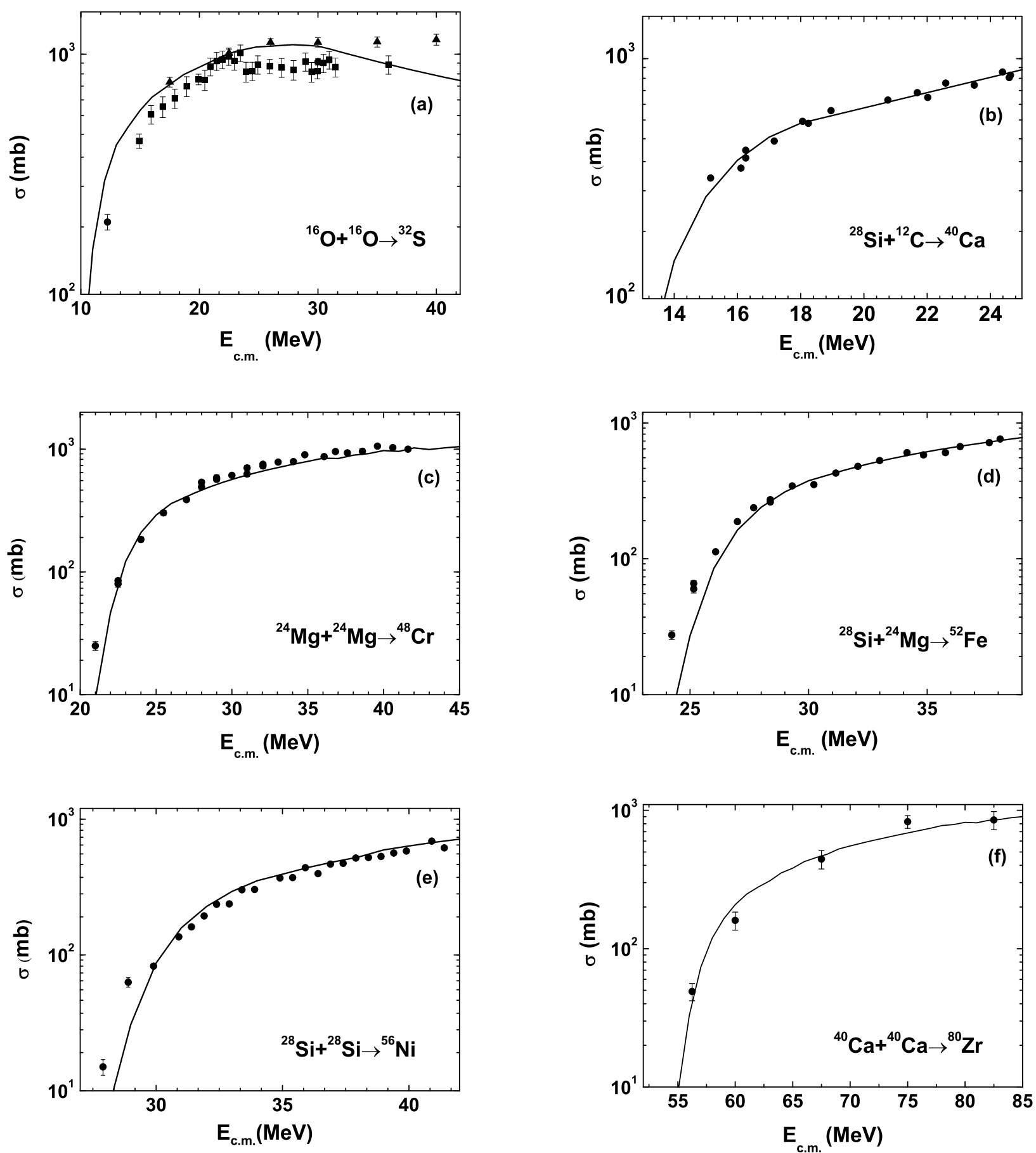

FIG. 2: Fusion cross sections around the Coulomb barrier for the ${ }^{16} \mathrm{O}+{ }^{16} \mathrm{O},{ }^{28} \mathrm{Si}+{ }^{12} \mathrm{C},{ }^{24} \mathrm{Mg}+{ }^{24} \mathrm{Mg},{ }^{28} \mathrm{Si}+{ }^{24} \mathrm{Mg},{ }^{28} \mathrm{Si}+{ }^{28} \mathrm{Si}$ and ${ }^{40} \mathrm{Ca}+{ }^{40} \mathrm{Ca}$ nuclear reactions.

minima appear at very high angular momenta. Their energetic and geometrical characteristics are provided as well as analytical formulas giving their energy as function of the angular momentum and the moment of inertia of the highest state. The barriers against decay into two fragments and against a direct descent to the compound nucleus are wide and high. New experiments using a beam energy in this energy range are desirable to study the possibility 
TABLE II: $l, \mathrm{I}, \mathrm{Q}, \beta$ and $\mathrm{E}$ are respectively the angular momentum, the moment of inertia, the quadrupole moment $\mathrm{Q}$, the $\beta$ parameter and the center of mass energy (relatively to the energy of two infinitely separated nuclei) of the strongly deformed quasi-molecular minima for symmetric fusion reactions between light and medium nuclei. B and $\mathrm{R}$ are the $l$-dependent fusion barrier heights and positions.

\begin{tabular}{|c|c|c|c|c|c|c|c|c|c|c|c|c|c|c|}
\hline Reaction & $l(\hbar)$ & $\mathrm{I}\left(\hbar^{2} / \mathrm{MeV}\right)$ & $\mathrm{Q}(\mathrm{e} b)$ & $\beta$ & $\mathrm{E}(\mathrm{MeV})$ & $\mathrm{B}(\mathrm{MeV})$ & $\mathrm{R}(\mathrm{fm})$ & $l(\hbar)$ & $\mathrm{I}\left(\hbar^{2} / \mathrm{MeV}\right)$ & $\mathrm{Q}(\mathrm{e} b)$ & $\beta$ & $\mathrm{E}(\mathrm{MeV})$ & $\mathrm{B}(\mathrm{MeV})$ & $\mathrm{R}(\mathrm{fm})$ \\
\hline${ }^{13} C+{ }^{13} C$ & 12 & 4.31 & 1.0 & 0.78 & 1.7 & 13.2 & 7.2 & 15 & 4.85 & 1.2 & 0.88 & 10.8 & 17.7 & 6.9 \\
\hline${ }^{13} C+{ }^{13} C$ & 18 & 5.34 & 1.4 & 0.94 & 20.8 & 23.5 & 6.5 & & & & & & & \\
\hline${ }^{16} \mathrm{O}+{ }^{16} \mathrm{O}$ & 12 & 4.45 & 0.6 & 0.39 & 2.13 & 15.7 & 7.7 & 15 & 6.43 & 1.7 & 0.82 & 7.76 & 18.8 & 7.5 \\
\hline${ }^{16} \mathrm{O}+{ }^{16} \mathrm{O}$ & 20 & 7.39 & 2.1 & 0.92 & 20.75 & 25.9 & 7.1 & 23 & 7.81 & 2.3 & 0.96 & 29.4 & 31.6 & 6.8 \\
\hline${ }^{24} \mathrm{Mg}+{ }^{24} \mathrm{Mg}$ & 22 & 11.1 & 2.4 & 0.68 & 15.4 & 31.8 & 8.25 & 25 & 11.9 & 2.9 & 0.76 & 21.7 & 34.8 & 8.1 \\
\hline${ }^{24} \mathrm{Mg}+{ }^{24} \mathrm{Mg}$ & 30 & 13.45 & 3.6 & 0.86 & 32.7 & 40.8 & 7.9 & 35 & 14.75 & 4.1 & 0.92 & 44.4 & 48.2 & 7.6 \\
\hline${ }^{24} \mathrm{Mg}+{ }^{24} \mathrm{Mg}$ & 37 & 15.1 & 4.3 & 0.94 & 49.3 & 51.6 & 7.5 & & & & & & & \\
\hline${ }^{28} S i+{ }^{28} S i$ & 27 & 14.2 & 3.1 & 0.67 & 24.3 & 40.9 & 8.5 & 30 & 15.3 & 3.6 & 0.75 & 30.2 & 43.8 & 8.4 \\
\hline${ }^{28} S i+{ }^{28} S i$ & 40 & 18.5 & 5.1 & 0.90 & 51.1 & 56.0 & 8.0 & 44 & 19.5 & 5.5 & 0.93 & 60.0 & 62.2 & 7.75 \\
\hline${ }^{40} \mathrm{Ca}+{ }^{40} \mathrm{Ca}$ & 38 & 25.9 & 5.5 & 0.65 & 52.7 & 69.3 & 9.2 & 40 & 26.9 & 6.0 & 0.70 & 55.7 & 70.8 & 9.2 \\
\hline${ }^{40} \mathrm{Ca}+{ }^{40} \mathrm{Ca}$ & 50 & 31.55 & 8.2 & 0.84 & 71.2 & 79.5 & 9.0 & 60 & 35.4 & 9.9 & 0.92 & 87.8 & 90.9 & 8.7 \\
\hline${ }^{40} \mathrm{Ca}+{ }^{40} \mathrm{Ca}$ & 62 & 35.9 & 10.15 & 0.93 & 91.3 & 93.5 & 8.6 & & & & & & & \\
\hline${ }^{48} \mathrm{Ca}+{ }^{48} \mathrm{Ca}$ & 48 & 34.2 & 5.7 & 0.61 & 47.8 & 68.4 & 9.8 & 50 & 35.4 & 6.3 & 0.65 & 50.6 & 69.8 & 9.8 \\
\hline${ }^{48} \mathrm{Ca}+{ }^{48} \mathrm{Ca}$ & 60 & 40.1 & 8.2 & 0.78 & 65.3 & 77.7 & 9.6 & 70 & 45.15 & 10.1 & 0.88 & 80.6 & 87.3 & 9.4 \\
\hline${ }^{48} \mathrm{Ca}+{ }^{48} \mathrm{Ca}$ & 80 & 49.3 & 11.6 & 0.93 & 96.6 & 98.8 & 9.1 & & & & & & & \\
\hline${ }^{58} N i+{ }^{58} N i$ & 49 & 51.0 & 11.1 & 0.70 & 99.2 & 113.5 & 10.1 & 50 & 51.55 & 11.3 & 0.71 & 100.2 & 114.0 & 10.1 \\
\hline${ }^{58} \mathrm{Ni}+{ }^{58} \mathrm{Ni}$ & 60 & 57.6 & 14.2 & 0.82 & 110.5 & 120.0 & 10.0 & 70 & 63.2 & 16.6 & 0.89 & 121.3 & 127.2 & 9.9 \\
\hline${ }^{58} N i+{ }^{58} N i$ & 80 & 67.6 & 18.4 & 0.92 & 132.8 & 135.7 & 9.7 & 83 & 68.8 & 18.9 & 0.94 & 136.5 & 138.5 & 9.6 \\
\hline
\end{tabular}

TABLE III: Angular momentum $l$, moment of inertia I and center of mass energy (in MeV and relatively to the energy of two infinitely separated nuclei) of the strongly deformed quasi-molecular minima for asymmetric fusion reactions between light and medium nuclei. $\mathrm{B}$ and $\mathrm{R}$ are the $l$-dependent fusion barrier heights and positions.

\begin{tabular}{|c|c|c|c|c|c|c|c|c|c|c|c|c|c|c|c|}
\hline Reaction & $l$ & $\mathrm{I}$ & $\mathrm{E}$ & $\mathrm{B}$ & $\mathrm{R}$ & $l$ & $\mathrm{I}$ & $\mathrm{E}$ & $\mathrm{B}$ & $\mathrm{R}$ & $l$ & $\mathrm{I}$ & $\mathrm{E}$ & $\mathrm{B}$ & $\mathrm{R}$ \\
\hline${ }^{28} \mathrm{Si}+{ }^{12} \mathrm{C}$ & 16 & 8.5 & 8.21 & 21.2 & 7.9 & 18 & 8.78 & 12.25 & 23.4 & 7.8 & 20 & 9.14 & 16.5 & 25.9 & 7.65 \\
\hline${ }^{28} \mathrm{Si}+{ }^{12} \mathrm{C}$ & 25 & 9.81 & 28.7 & 33.6 & 7.3 & 28 & 10.15 & 36.8 & 39.3 & 7.15 & & & & & \\
\hline${ }^{28} \mathrm{Si}+{ }^{16} \mathrm{O}$ & 17 & 9.86 & 9.34 & 24.5 & 8.15 & 20 & 10.59 & 14.95 & 27.3 & 8.0 & 25 & 11.7 & 25.4 & 33.2 & 7.8 \\
\hline${ }^{28} \mathrm{Si}+{ }^{16} \mathrm{O}$ & 30 & 12.4 & 37.0 & 40.8 & 7.5 & 32 & 12.7 & 42.1 & 44.4 & 7.3 & & & & & \\
\hline${ }^{28} \mathrm{Si}+{ }^{24} \mathrm{Mg}$ & 24 & 10.0 & 19.8 & 35.7 & 8.4 & 25 & 13.0 & 20.9 & 36.6 & 8.35 & 30 & 19.5 & 30.5 & 41.9 & 8.2 \\
\hline${ }^{28} \mathrm{Si}+{ }^{24} \mathrm{Mg}$ & 35 & 15.9 & 42.0 & 48.3 & 8.0 & 40 & 17.2 & 53.5 & 56.0 & 7.75 & & & & & \\
\hline${ }^{28} \mathrm{Si}+{ }^{40} \mathrm{Ca}$ & 31 & 19.95 & 35.6 & 52.1 & 8.9 & 35 & 21.5 & 42.1 & 55.5 & 8.8 & 40 & 23.1 & 50.6 & 60.3 & 8.7 \\
\hline${ }^{28} \mathrm{Si}+{ }^{40} \mathrm{Ca}$ & 45 & 25.0 & 59.5 & 66.0 & 8.5 & 50 & 26.2 & 68.9 & 72.5 & 8.3 & 53 & 27.1 & 74.1 & 76.8 & 8.2 \\
\hline${ }^{40} \mathrm{Ca}+{ }^{48} \mathrm{Ca}$ & 52 & 30.0 & 57.1 & 76.1 & 9.4 & 55 & 31.55 & 62.4 & 78.8 & 9.3 & 60 & 33.6 & 71.4 & 70.8 & 9.2 \\
\hline${ }^{40} \mathrm{Ca}+{ }^{48} \mathrm{Ca}$ & 65 & 35.7 & 80.45 & 89.25 & 9.15 & 70 & 37.9 & 89.7 & 95.4 & 8.8 & 75 & 40.0 & 99.05 & 102.3 & 8.3 \\
\hline${ }^{40} \mathrm{Ca}+{ }^{48} \mathrm{Ca}$ & 78 & 41.2 & 104.8 & 107.0 & 8.25 & & & & & & & & & & \\
\hline
\end{tabular}

to form these hyperdeformed rotating states.

[1] C. Beck et al., Acta Phys. Pol. B 42, 747 (2011).

[2] W. von Oertzen et al., Eur. Phys. J. A 36, 279 (2008).

[3] W. von Oertzen, V. Zherebchevsky, B. Gebauer, Ch. Schulz, S. Thummerer, D. Kamanin, G. Royer, and Th. Wilpert, Phys. Rev. C 78, 044615 (2008).

[4] E. Ideguchi et al., Phys. Rev. Lett. 87, 222501 (2001).

[5] C.E. Svensson et al., Phys. Rev. C 63, 061301 (R) (2001).

[6] S.J. Sanders et al., Phys. Rep. 311, 487 (1999).

[7] S. Yu. Kun, B.A. Robson and A.V. Vagov, Phys. Rev. Lett. 83, 504 (1999).

[8] C. Beck et al., Phys. Rev. C 63, 014607 (2000).

[9] M. Rousseau et al., Phys. Rev. C 66, 034612 (2002). 
TABLE IV: $l$-dependent fusion barrier height $\mathrm{B}$ (in $\mathrm{MeV}$ ) and position $\mathrm{R}$ (in $\mathrm{fm}$ ) for fusion reactions between light and medium nuclei.

\begin{tabular}{|c|c|c|c|c|c|c|c|c|c|c|c|c|}
\hline Reaction & $l(\hbar)$ & $B(l)$ & $R(l)$ & $l(\hbar)$ & $B(l)$ & $R(l)$ & $l(\hbar)$ & $B(l)$ & $R(l)$ & $l(\hbar)$ & $B(l)$ & $R(l)$ \\
\hline${ }^{13} C+{ }^{13} C$ & 0 & 5.85 & 8.10 & 3 & 6.4 & 8.0 & 6 & 7.9 & 7.75 & 9 & 9.9 & 7.45 \\
\hline${ }^{16} \mathrm{O}+{ }^{16} \mathrm{O}$ & 0 & 10.3 & 8.2 & 5 & 11.3 & 8.2 & 10 & 14.0 & 7.8 & & & \\
\hline${ }^{28} \mathrm{Si}+{ }^{12} \mathrm{C}$ & 0 & 13.2 & 8.40 & 5 & 14.0 & 8.35 & 10 & 16.3 & 8.25 & 15 & 20.2 & 7.9 \\
\hline${ }^{28} \mathrm{Si}+{ }^{16} \mathrm{O}$ & 0 & 17.3 & 8.55 & 5 & 18.0 & 8.5 & 10 & 19.8 & 8.4 & 15 & 22.9 & 8.2 \\
\hline${ }^{24} \mathrm{Mg}+{ }^{24} \mathrm{Mg}$ & 0 & 21.9 & 8.7 & 5 & 22.4 & 8.7 & 10 & 24.0 & 8.6 & 15 & 26.5 & 8.5 \\
\hline${ }^{24} \mathrm{Mg}+{ }^{24} \mathrm{Mg}$ & 20 & 30.1 & 8.3 & & & & & & & & & \\
\hline${ }^{28} \mathrm{Si}+{ }^{24} \mathrm{Mg}$ & 0 & 25.2 & 8.8 & 5 & 25.7 & 8.8 & 10 & 27.1 & 8.7 & 15 & 29.3 & 8.65 \\
\hline${ }^{28} \mathrm{Si}+{ }^{24} \mathrm{Mg}$ & 20 & 32.5 & 8.5 & & & & & & & & & \\
\hline${ }^{28} \mathrm{Si}+{ }^{28} \mathrm{Si}$ & 0 & 29.0 & 8.95 & 10 & 30.7 & 8.9 & 20 & 35.5 & 8.7 & & & \\
\hline${ }^{28} \mathrm{Si}+{ }^{40} \mathrm{Ca}$ & 0 & 40.2 & 9.25 & 10 & 41.5 & 9.2 & 20 & 45.1 & 9.1 & 30 & 51.4 & 8.9 \\
\hline${ }^{40} \mathrm{Ca}+{ }^{40} \mathrm{Ca}$ & 0 & 55.6 & 9.6 & 10 & 56.6 & 9.55 & 20 & 59.4 & 9.5 & 30 & 64.1 & 9.4 \\
\hline${ }^{40} \mathrm{Ca}+{ }^{48} \mathrm{Ca}$ & 0 & 54.0 & 9.9 & 10 & 54.8 & 9.9 & 20 & 57.2 & 9.8 & 30 & 61.2 & 9.7 \\
\hline${ }^{40} \mathrm{Ca}+{ }^{48} \mathrm{Ca}$ & 40 & 66.9 & 9.6 & 50 & 74.4 & 9.4 & & & & & & \\
\hline${ }^{48} \mathrm{Ca}+{ }^{48} \mathrm{Ca}$ & 0 & 52.4 & 10.2 & 10 & 56.6 & 10.2 & 20 & 55.2 & 10.15 & 30 & 58.6 & 10.1 \\
\hline${ }^{48} \mathrm{Ca}+{ }^{48} \mathrm{Ca}$ & 40 & 63.4 & 9.95 & & & & & & & & & \\
\hline${ }^{58} \mathrm{Ni}+{ }^{58} \mathrm{Ni}$ & 0 & 100.6 & 10.4 & 10 & 100.2 & 10.4 & 20 & 102.8 & 10.3 & 30 & 105.4 & 10.3 \\
\hline${ }^{58} \mathrm{Ni}+{ }^{58} \mathrm{Ni}$ & 40 & 109.2 & 10.2 & & & & & & & & & \\
\hline
\end{tabular}

[10] A. Galindo-Uribarri et al., Phys. Rev. Lett. 71, 231 (1993).

[11] D.R. LaFosse et al., Phys. Rev. Lett. 74, 5186 (1995).

[12] D.R. LaFosse et al., Phys. Rev. C 54, 1585 (1996).

[13] J.M. Nieminen et al., Phys. Rev. C. 58, R1 (1998).

[14] E.S. Paul et al., Phys. Rev. Lett. 98, 012501 (2007).

[15] G. Giardina, F. Hanappe, A.I. Muminov, A.K. Nasirov, and L. Stuttgé, Nucl. Phys. A 671, 165 (2000).

[16] G. Fazio et al., Phys. Rev. C 72, 064614 (2005).

[17] A.K. Nasirov, G. Mandaglio, M. Manganaro, A.I. Muminov, G. Fazio, and G. Giardina, Phys. Lett. B 686, 72 (2010).

[18] G. Royer and F. Haddad, Phys. Rev. C 47, 1302 (1993).

[19] G. Royer and B. Remaud, Nucl. Phys. A 444, 477 (1985).

[20] G. Royer, J. Phys. G: Nucl. Phys. 12, 623 (1986).

[21] G. Royer, C. Bonilla and R.A. Gherghescu, Phys. Rev. C 67, 034315 (2003).

[22] G. Royer and C. Piller, J. Phys. G : Nucl. Part. Phys. 18, 1805 (1992).

[23] G. Royer, J. Phys. G : Nucl. Part. Phys. 26, 1149 (2000).

[24] G. Royer and R. Moustabchir, Nucl. Phys. A 683, 182 (2001).

[25] A.S. Zubov, V.V. Sargsyan, G.G. Adamian, N.V. Antonenko and W. Scheid, Phys. Rev. C 82, 034610 (2010).

[26] L.C. Vaz, J.M. Alexander and G.R. Satchler, Phys. Rep. 69, 373 (1981).

[27] J.R. Birkelund and J.R. Huizenga, Ann. Rev. Nucl. Part. Sci. 33, 265 (1983).

[28] S. Gary and C. Volant, Phys. Rev. C 25, 1877 (1982).

[29] E. Tomasi, D. Ardouin, J. Barreto, V. Bernard, B. Cauvin, C. Magnago, C. Mazur, C. Ngo, E. Piasecki, and M. Ribrag, Nucl. Phys. A 373, 341 (1982).

[30] M. Trotta, A.M. Stefanini, L. Corradi, A. Gadea, F. Scarlassara, S. Beghini, and G. Montagnoli, Phys. Rev. C 65, 011601(R) (2001). 